
\title{
25 Research Square \\ Rotational Stability of Proximally Unlocked \\ Retrograde Femoral Nail In Damage Control Surgery \\ - Biomechanical Study
}

Rahil Muzaffar ( $\sim$ rahil@squ.edu.om )

Sultan Qaboos University Hospital https://orcid.org/0000-0003-4828-4594

Muadh Hamood Nasser Al Zeedi

Sultan Qaboos University Hospital

Khurshid Alam

Sultan Qaboos University

Ahmed Yaseen

Sultan Qaboos University Hospital Muscat Oman

Sultan Al Maskari

Sultan Qaboos University Hospital

\section{Research Article}

Keywords: Unlocked intramedullary nailing, Damage control orthopaedics, Femoral shaft fractures, Temporary external fixation.

Posted Date: December 8th, 2021

DOI: https://doi.org/10.21203/rs.3.rs-1002602/v1

License: (c) (1) This work is licensed under a Creative Commons Attribution 4.0 International License. Read Full License 


\section{Abstract}

BACKGROUND: This biomechanical study was performed to look into the rotational stability of retrograde femoral intramedullary nail when it is used without proximal locking as a damage control device for management of femoral shaft fractures in emergency situations. This study compares this technique with the accepted methods for femoral shaft fixations in damage control surgeries. An alternative technique of using lateral compression screw to provide additional rotational stability is described.

METHODS: Experiments were divided into four different sets. Distally locked retrograde nail was passed across the fracture without any proximal fixation in set 1 , a compression screw passed from lateral cortex in set 2 , a proximal locking screw fixation in set 3 . In set 4 , Saw bone was fixed with external fixator.

The lateral compression screw group was further sub divided into three subgroups based on the amount of torque applied manually.

The torsion test was applied to create an rotational displacement of 10 degrees and the maximum load required to create the rotational displacement was noted.

RESULTS: Application of a compression screw improved the rotational stability significantly in comparison to no proximal locking. In the subgroup III of lateral compression screw application, the rotational stability was found to be equivalent to stability achieved with Nail with proximal locking and was found to be greater in comparison to external fixator application.

CONCLUSION: This study shows that the addition of a lateral compression screw significantly improves rotational stability and has the potential to be used in emergency lifesaving procedures.

\section{Background}

Proximally and distally locked intramedullary nailing (IMN) of femoral shaft fractures is accepted as the standard treatment as part of early total care (ETC) in the hemodynamically stable patient. Unfortunately, in hemodynamically unstable patients, this technique has been associated with greater morbidity and mortality $[1,2,3]$. Hence calls for less aggressive fixation methods were made. Currently, the use of external fixators as temporary fixation of these fractures as part of damage control orthopaedics (DCO) in these patients is accepted as the treatment of choice $[2,4,5]$.

However, external fixation (EF) in these patients is far from ideal. It affords only partial fracture stability especially in the obese thigh with potential for medullary canal content embolization. Pin site infection is another major drawback that has significant bearing on future management options. Knee stiffness because of soft tissue splinting is a third problem. Nursing in intensive care units (ICU), especially if they have bilateral $\mathrm{EF}$, is a major problem in these patients who require regular turning and physiotherapy. Other complications include continued bleeding from the fracture site and rare vascular injuries (6). 
Finally, conversion to standard locked IMN nail is a major procedure that maybe associated with further complications and additional costs $(7,8,9,10)$.

Recent studies have suggested the use of retrograde intramedullary femoral nail (RIMFN) as an alternative to EF as damage control device $(11,12)$. A small diameter nail is passed gently without reaming and is only locked distally. Proximal locking is done at later stage once the patient's condition has stabilized. This technique has been found to be relatively safe, easy and quick. There is no need for a second major surgical procedure thereby minimizing the chance of the second hit associated with later IMN.

However, there are concerns with regards to the rotational stability of the fracture fixation where RIMFN has been used without proximal locking. One option is adding a lateral compression screw proximally, to improve the rotational stability of the construct.

To the authors knowledge there are no studies of the biomechanical performance of RIMFN of shaft fractures without proximal locking in terms of rotational stability.

The current experimental investigation was performed to evaluate the role of a proximal lateral compression screw in rotational stability compared to no proximal fixation and standard proximally locked nail. The rotational stability of nail was also compared with External fixation for this injury.

\section{Methods}

Twelve fourth generation Sawbones (made of fiber filled epoxy cortical shell and cancellous polyurethane core to mimic young human adult healthy femurs) measuring $455 \mathrm{~mm}$ in length and reamed to an internal diameter of $13 \mathrm{~mm}$ (Sawbones Worldwide / Pacific Research Laboratories, Malmö, Sweden) were used. M/DN Femoral Retrograde Intramedullary nails (Zimmer-Biomet, Warsaw, Indiana, USA) length 380mm, and diameter $10 \mathrm{~mm}$, proximal screw size $4.2 \mathrm{~mm}$, distal screw size $5.5 \mathrm{~mm}$ were used.

The femur was potted in a mounting fixture containing two customised iron boxes on either end and placed on a servo-hydraulic testing machine (SM1 Mkll Torsion Testing Machine) for mechanical testing (Fig 1).

A torsion force was applied to create an rotational displacement of 10 degrees. No load was exerted axially along the femur during torsion testing, thus avoiding frictional resistance at the fracture site. The load/Torque applied was measured in Newton meters $(\mathrm{Nm})$ using digital torque measuring machine (TeCQuipment E -101 Digital Measuring System)

Experiments were divided into four different sets of three saw bones each.

Group 1: Distally locked RIMFN passed across the fracture without any proximal fixation 
Group 2: Distally locked RIMFN passed across the fracture with a $4.2 \mathrm{~mm}$ blocking bolt (lateral compression screw) was passed through the lateral cortex to compress the nail against the medial cortex (Fig.2).

Group 3: Distally locked RIMFN passed across the fracture with one $4.2 \mathrm{~mm}$ anterior to posterior proximal locking screw.

Group 4: Saw bone was fixed with external fixators, using two Schanz's screws in each fragment.

Each set included three saw bones and for each mode of fixation the biomechanical testing was performed three times.

The nail was inserted through the intercondylar notch using the manufacturer's recommended surgical technique. The nail-head was countersunk $2.0 \mathrm{~mm}$ relative to the base of the intercondylar notch. Two distal locking screws were inserted using the nail-mounted drill guide. A transverse osteotomy was performed $18 \mathrm{~cm}$ above the articular surface ensuring free movement of the proximal fragment in relation the distal fragment-nail composite.

The lateral compression screw $(4.2 \mathrm{~mm})$ was passed through the lateral cortex at proximal end of the nail (after predrilling the bone with $3.5 \mathrm{~mm}$ drill) which was identified using an $\mathrm{X}$ ray (Fig 2). The torsion force required to introduce the screw was increased progressively in three sub groups of saw bones. In sub group I, after the screw was felt to come in contact with the nail in medullary canal, the screw was advanced for around two to three complete turns. In the second set of saw bones (subgroup II) the screw was advanced further for approximately three turns till the force required to advance the screw was felt to be sufficient to create enough force on the nail to prevent any rotatory moment of nail in the canal. In the third group (sub group III), the torque was applied to the screw till it could not be advanced any further.

The unilateral uniplanar fixator (Sharma Ortho System Pvt Limited, India) was used. Two 6 $\mathrm{mm}$ Schanz's pins were inserted bicortically in each of the proximal and distal fragments. The core drillholes were $4.5 \mathrm{~mm}$. The pins were connected using a single rod of $11 \mathrm{~mm}$ diameter using rod to pin clamps.

Statistical analysis using independent paired T test with significance level of $P<0.05$ was performed.

\section{Results}

Table 1 shows the results of each individual reading.

\section{Table 1: Results of biomechanical tests (torque in Nm).}




\begin{tabular}{|lllll|}
\hline & Nail (NPL) & Nail (B) & Nail (PL) & Ex Fix \\
\hline Saw bone 1 & 0.21 & 0.58 & 1.66 & 0.95 \\
\cline { 2 - 5 } & 0.20 & 0.86 & 1.53 & 0.92 \\
\cline { 2 - 5 } & 0.18 & 0.96 & 1.60 & 0.95 \\
\hline Saw bone 2 & 0.17 & 0.74 & 1.86 & 0.93 \\
\cline { 2 - 5 } & 0.20 & 0.95 & 1.50 & 0.85 \\
\hline Saw bone 3 & 0.17 & 0.90 & 1.59 & 0.85 \\
\cline { 2 - 5 } & 0.21 & 1.50 & 1.08 & 0.74 \\
\cline { 2 - 5 } & 0.19 & 1.43 & 1.09 & 0.71 \\
\hline Mean & 0.1922 & 1.037 & 1.4444 & 0.8333 \\
\hline
\end{tabular}

Nail (NPL): Nail with no proximal locking.Nail (B): Nail with bolt (lateral compression screw).Nail (PL): Nail with proximal locking. Ex Fix: External fixator.

The mean torsional loads required to create 10 degrees of rotational deformity in set 1,2,3 and 4 was $0.19,1.03,1.44$ and 0.83 , respectively. The standard deviation and standard error for each group are shown in table 2.

Table: 2. Mean Torque required to create 10 degrees of rotational displacement for each group.

\begin{tabular}{|lllll|}
\hline & N & $\begin{array}{l}\text { Mean } \\
\text { (Torque Nm) }\end{array}$ & Std. Deviation & Std. Error Mean \\
\hline Nail (NPL) & 9 & 0.1922 & 0.01563 & 0.00521 \\
& & & & \\
Nail(B) & 9 & 1.037 & 0.3295 & 0.1098 \\
& & & & \\
Nail (PL) & 9 & 1.4444 & 0.2870 & 0.0957 \\
& & & & 0.0413 \\
\hline Ex Fix & 9 & 0.8333 & 0.1240 & \\
\hline
\end{tabular}

Nail (NPL): Nail with no proximal locking.Nail (B): Nail with bolt (lateral compression screw).Nail $(\mathrm{PL})$ : Nail with proximal locking. Ex Fix: External fixator. 
Torsional stability varied between the different groups. As expected, the nail without any proximal screws was found to be the least stable construct (Fig. 3). Application of lateral compression bolt improved the rotational stability significantly in comparison to no proximal locking (Fig. 4). Rotational stability with lateral compression screw was found to be higher than the external fixator group but the difference was not found to be statistically significant (Table 3 ).

\section{Table 3: Comparative analysis of all groups.}

\begin{tabular}{|llll|}
\hline & M & Cl & Sig (2-tailed) \\
\hline Pair 1 & 0.8444 & 0.6113 to 1.0775 & 0.00 \\
\hline Pair 2 & 0.2033 & -0.454 to 0.4521 & 0.102 \\
\hline Pair 3 & 0.6411 & 0.5528 to 0.7294 & 0.00 \\
\hline Pair 4 & 0.6111 & 0.3902 to 0.8320 & 0.00 \\
\hline Pair 5 & 0.4078 & 0.0990 to 0.7166 & 0.01 \\
\hline
\end{tabular}

Pair 1: Nail with Bolt (lateral compression screw) vs Nail without proximal screw.Pair 2: Nail with Bolt (lateral compression screw) vs External FixatorPair 3: External fixator vs Nail without proximal screwPair 4: Proximal locking screw vs External fixatorPair 5: Nail with Bolt (lateral compression screw) vs Proximal locking screwM: Difference of means.Cl: $95 \%$ Confidence Interval of difference in means.

Nail with proximal locking screw was found to be most stable rotationally.

On assessment of subgroups in the lateral compression screw group, the means in subgroup I, II and III were $0.800,0.863$ and 1.447 respectively (Table 4).

Table 4: Mean Torque ( $\mathrm{Nm})$ in lateral compression screw sub groups

\begin{tabular}{|lc|}
\hline Sub group & Mean Torque required for 10degrees of rotational displacement \\
\hline I & 0.800 \\
\hline II & 0.863 \\
III & 1.447 \\
\hline
\end{tabular}

Mean torque in the subgroup III was found to be statistically higher in comparison to sub groups I and II $(P$-value $<.001)$. The subgroups were compared with external fixator group and with Nail with proximal locking group (Table 5).

Table 5: Comparison of Means of sub groups in lateral compression screw with External fixator and Nail with proximal locking 


\begin{tabular}{|ll|}
\hline Groups (Mean Torque in brackets) & $P$-value \\
\hline Sub group I (0.800) vs Ex Fix (0.833) & 0.73 \\
\hline Sub group I (0.800) vs Nail (PL) (1.444) & 0.00 \\
\hline Sub group II (0.863) vs Ex Fix (0.833) & 0.71 \\
\hline Sub group II (0.863) vs Nail (PL) & 0.00 \\
\hline Sub group III (1.447) vs Ex Fix (0.833) & 0.00 \\
\hline Sub group III (1.447) vs Nail (PL)(1.444) & 0.98 \\
\hline
\end{tabular}

Nail (PL): Nail with proximal locking. Ex Fix: External fixator

Results showed that the mean torque of subgroup III of lateral compression screw was highest of all groups but it was not statistically more in comparison to Nail with proximal locking. In comparison to external fixator group, sub group III was found to be significantly stable in terms of rotational stability.

\section{Discussion}

This study employed mechanical testing to evaluate the rotational stability of various constructs used in the treatment of femoral fractures in polytrauma scenarios. The results showed that rotational stability was best achieved using a distally and proximally locked retrograde femoral nail. However, this construct is contraindicated in the hemodynamically unstable polytrauma patient where speed and minimal surgical trauma are of vital importance. In these patients, the current standard of care is application of EF with 2 screws in each fragment $(4,5)$. This construct showed statistically significant lower rotational stability in comparison with the fully locked nail [Mean difference $0.61 ; 95 \% \mathrm{Cl}$ of difference $=0.39-0.83$; $P=0.00]$.

Traditionally external fixators have been used as damage control devices in polytrauma patients for femoral fixations. Recent studies of a modified protocol of retrograde nailing for damage control indicate it to be efficient, effective, and safe $(11,12)$. However, passing a nail without proximal locking makes it rotationally unstable which has been a concern against the use of RIMFN as damage control device.

In order to improve the rotational stability, the authors propose the use of screw passed from lateral cortex proximally as a bolt. The screw pushes the nail against the far (medial) cortex and minimizes rotation of the nail in the medullary canal. This technique significantly improves the rotational strength in comparison to no proximal fixation (Fig 4). The mean force required to create the rotational displacement was lower in external fixators in comparison to this technique but this difference was not found to be statistically significant (Table 3). However. when the individual subgroups in lateral screw group were compared with other groups, the subgroup III was found to have highest rotational stability (Fig 5) though the difference in comparison to nail with proximal locking was found to have no statistical significance (Table 5). 
This biomechanical study has shown that using a proximal lateral compression screw provides significant rotational stability and is a quick and simple procedure that can be used to temporarily provide rotational stability in emergency situations. Further, it requires only a single $\mathrm{C}$ arm exposure to identify the proximal end of the nail thereby decreasing the surgical time and at the same time providing significantly improved stability.

\section{Abbreviations}

ETC - Early total care

IMN - Intramedullary nailing

DCO- Damage control orthopaedics

EF - External fixation

ICU- Intensive care units

RIMFN - Retrograde intramedullary femoral nail

\section{Declarations}

Ethics approval and consent to participate: Not applicable

Consent for publication: Not applicable

Availability of data and materials: All data generated or analysed during this study are included in this published article

Competing interests: The authors declare that they have no competing interests

Funding: This work was supported by Sultan Qaboos University Muscat Oman [RF/MED/SURG/19/01].

Acknowledgements: Not applicable

\section{Authors' contributions:}

RM: Involved in design and setting of the study.Performed biomechanical tests and collected the data, involved in writing of the manuscript

MHNZ: Did the background research and was involved in design and setting of the study

KA: Involved in designing the biomechanical tests protocol

AY: Involved in writing of the manuscript 
SAM: Involved in design and setting of the study. Involved in writing of the manuscript

\section{References}

1. Pape H-C, Grimme K, Van Griensven M, Sott AH, Giannoudis P, Morley J, et al. Impact of intramedullary instrumentation versus damage control for femoral fractures on immunoinflammatory parameters: prospective ran- domized analysis by the EPOFF Study Group. J Trauma 2003;55(1):7-13,

2. Pape HC, Rixen D, Morley J, Husebye EE, Mueller M, Dumont M et al. Impact of the Method of Initial Stabilization for Femoral Shaft Fractures in Patients with Multiple Injuries at Risk for Complications (Borderline Patients). Ann Surg. 2007 Sep; 246(3): 491-501

3. H. Nieto and C. Baroan. Limits of internal fixation in long-bone fracture. Orthopaedics \& Traumatology: Surgery \& Research 2017 (103) S61-S66

4. Scalea TM, Boswell SA, Scott JD, Mitchell KA, Kramer ME, Pollak AN. External fixation as a bridge to intramedullary nailing for patients with multiple injuries and with femur fractures: damage control orthopaedics. J Trauma. 2000 Apr;48(4):613-23.

5. James H. CARSON. Damage Control Orthopaedics - When and Why. Journal of Lancaster General Hospital. 2007; Vol. 2; No. 3:103-105

6. Staeheli GR, Fraser MR and Morgan SJ. The dangers of damage control orthopaedics: a case report of vascular injury after femoral fracture external fixation. Patient Safety in Surgery 2012, 6:7. Available http://www.pssjournal.com/content/6/1/7. Accessed on Oct,01,2018. Accessed May,28,2018.

7. Nowotarski PJ1, Turen CH, Brumback RJ and Scarboro JM. Conversion of external fixation to intramedullary nailing for fractures of the shaft of the femur in multiply injured patients. J Bone Joint Surg Am. 2000. Jun;82(6):781-8.

8. Stefan R, Ronny B, Tim W, Melanie G, Esther W, Florian G et al. Conversion from External Fixator to Intramedullary Nail Causes a Second Hit and Impairs Fracture Healing in a Severe Trauma Model. Journal of orthopaedic research. March 2013:465-71

9. Austin TF and Rozbruch SR. The Mechanics of External Fixation. HSSJ. 2007; 3: 13-29.

10. Harwood PJ1, Giannoudis PV, Probst C, Krettek C and Pape HC. The risk of local infective complications after damage control procedures for femoral shaft fracture. J Orthop Trauma. 2006 Mar;20(3):181-9.

11. Higgins Thomas F., Horwitz Daniel S. Damage control nailing. J. Orthop. Trauma. 2007 August;21(7):477-481

12. Retrograde femoral nails for emergency stabilization in multiply injured patients with haemodynamic instability. Sultan Al Maskari, Rahil Muzaffar, and Ahmed Yaseen. Trauma Case Rep. 2020 Oct; 29: 100350. 
Figures

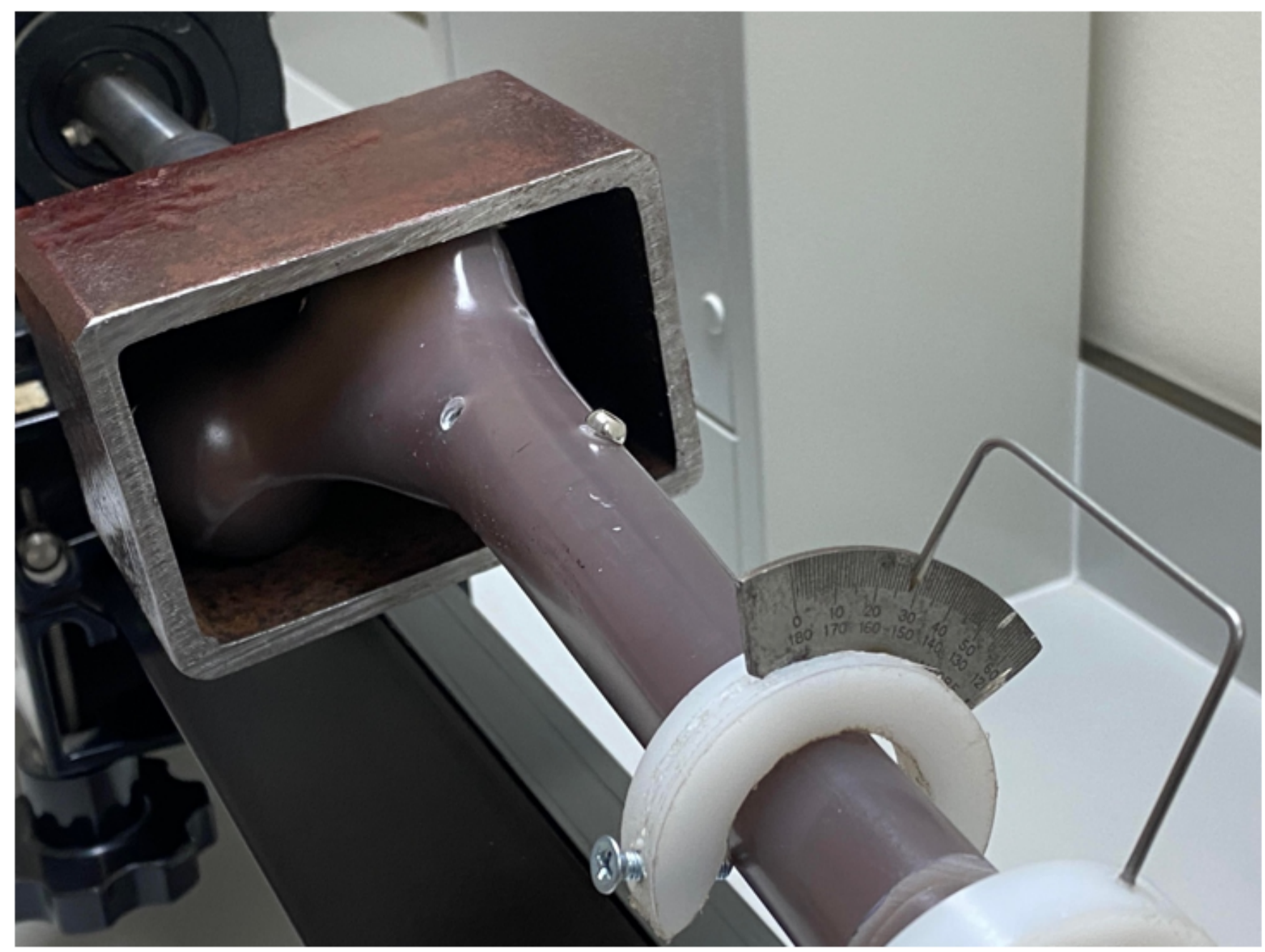

Figure 1

Demonstrating the customized mounting fixture for the nail ends. 


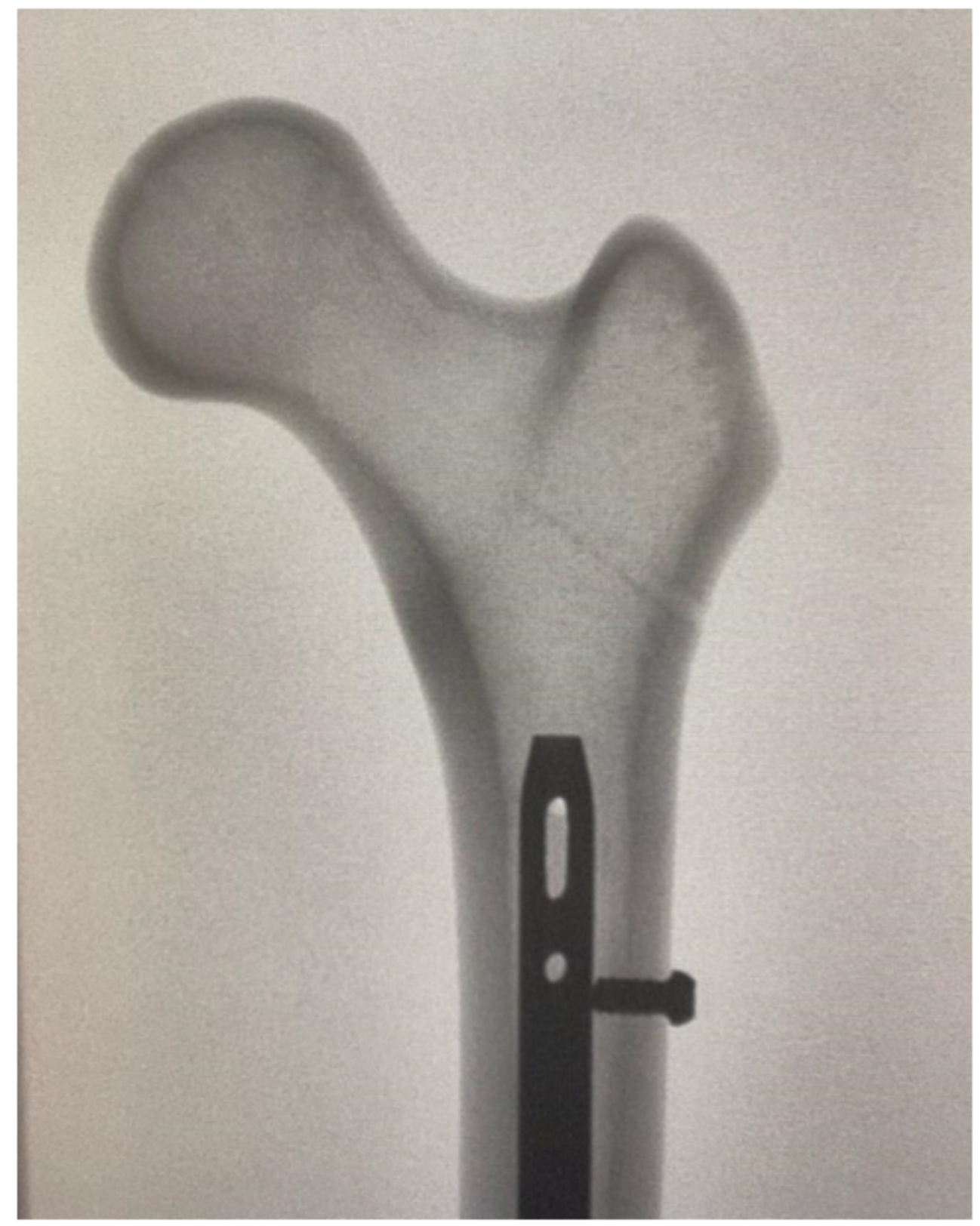

Figure 2

Image intensifier $\mathrm{X}$-ray demonstrating the lateral compression screw application. 


\section{Mean Torque ( $\mathrm{Nm})$}

1.8

1.6

1.4

1.2

1

0.8

0.6

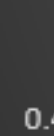

0.4

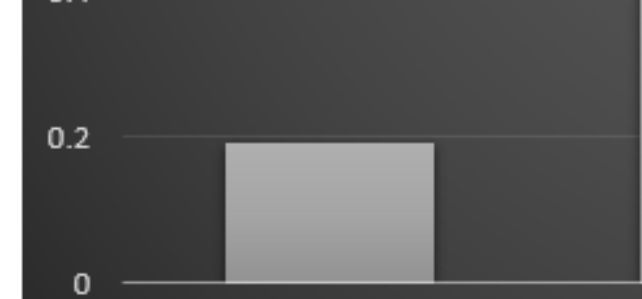

Nail (NPL)

Nail (B)

Nail (PL)

Ex Fix

\section{Figure 3}

Mean Torque Nail (NPL): Nail with no proximal locking Nail (B): Nail with bolt (lateral compression screw) Nail (PL): Nail with proximal locking Ex Fix: External fixator 


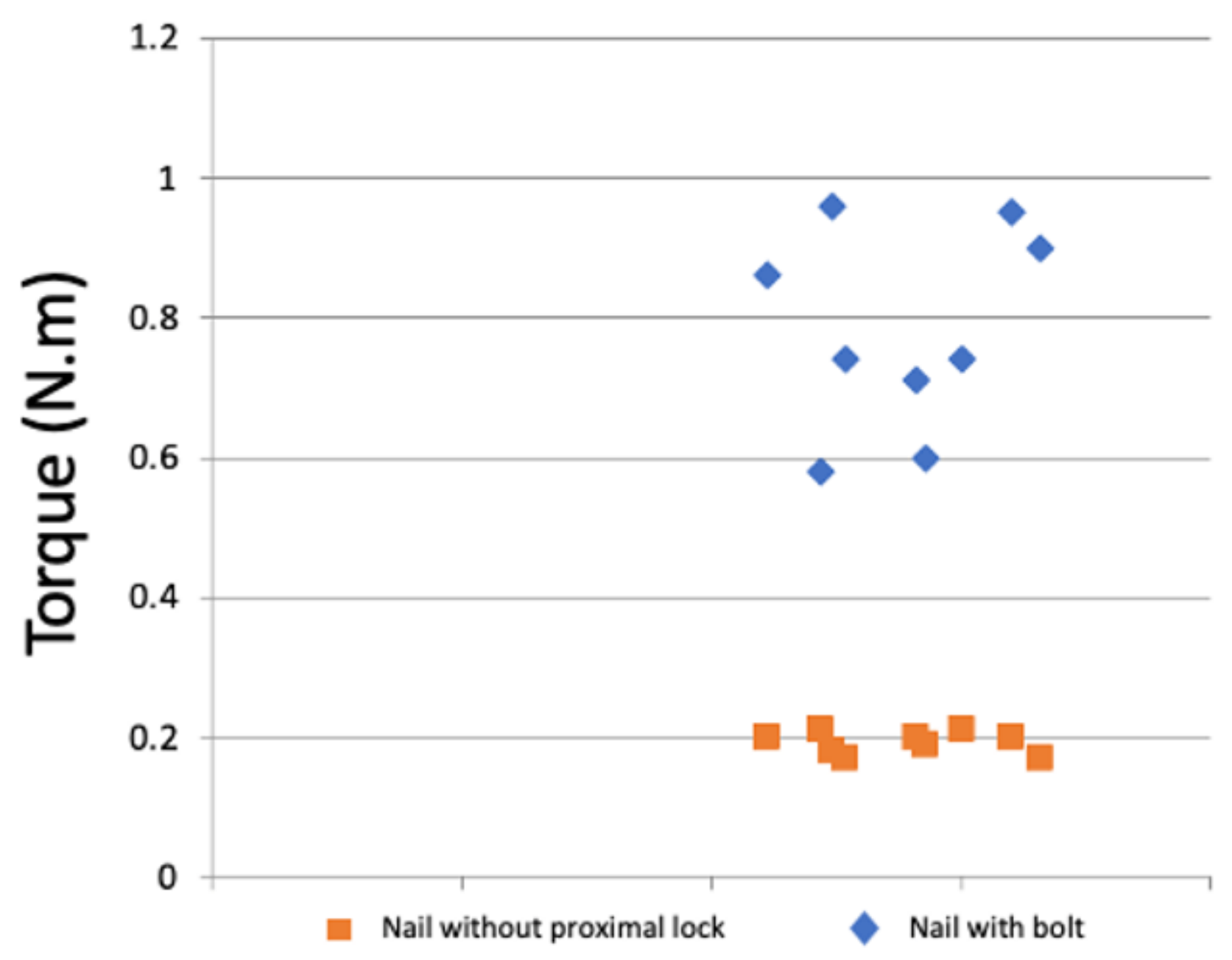

Figure 4

Scatter diagram depicting the torque loads for Nail with no proximal lock and Nail with bolt (lateral compression screw). N.m: Newton meters 


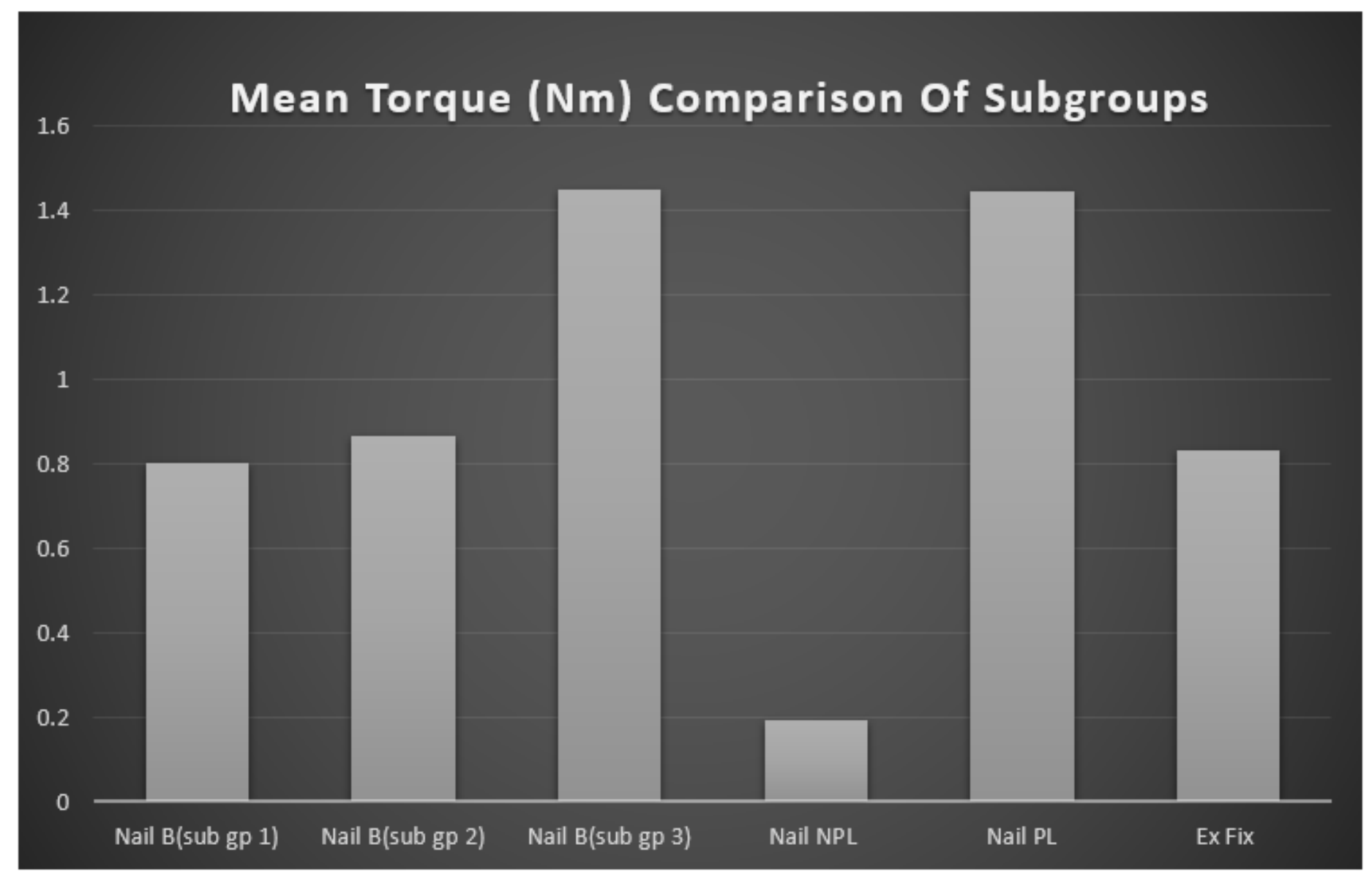

\section{Figure 5}

Demonstrating mean Torque values of subgroups in comparison to all groups Nail (NPL): Nail with no proximal locking. Nail (B): Nail with bolt (lateral compression screw). Nail (PL): Nail with proximal locking. Ex Fix: External fixator. 